\title{
Knowledge, risk perception and access to healthcare services for HIV and tuberculosis among university students in Johannesburg, South Africa
}

\author{
D Evans, ${ }^{1}$ DBiomed; N Musakwa, ${ }^{1} \mathrm{MPH}$; C Nattey, ${ }^{1} \mathrm{MSc}$; J Bor, ${ }^{2}$ ScD SM; E Lönnermark, ${ }^{3} \mathrm{PhD}, \mathrm{MD} ; \mathrm{C}$ Larshans, ${ }^{3} \mathrm{MB}$ BCh; \\ S Andreasson, ${ }^{3} \mathrm{MB}$ BCh; P Nyasulu, ${ }^{4,5} \mathrm{PhD}$ Epi; L Long, ${ }^{1,2} \mathrm{PhD}$ \\ ${ }^{1}$ Health Economics and Epidemiology Research Office, Department of Internal Medicine, School of Clinical Medicine, Faculty of Health Sciences, \\ University of the Witwatersrand, Johannesburg, South Africa \\ ${ }^{2}$ Department of Global Health, Boston University School of Public Health, USA \\ ${ }^{3}$ Department of Infectious Diseases, Sahlgrenska University Hospital, Gothenburg, Sweden \\ ${ }^{4}$ Division of Epidemiology and Biostatistics, Department of Global Health, Faculty of Medicine and Health Sciences, Stellenbosch University, \\ Cape Town, South Africa \\ ${ }^{5}$ Division of Epidemiology and Biostatistics, School of Public Health, Faculty of Health Sciences, University of the Witwatersrand, Johannesburg, \\ South Africa
}

Corresponding author: D Evans (devans@heroza.org)

\begin{abstract}
Background. An increasingly diverse body of students is entering university in South Africa. HIV and tuberculosis (TB) are pressing health issues for this vulnerable population and the university campus offers an opportunity to intervene with health promotion activities. Objectives. This study describes knowledge and risk perception of TB and HIV among high school leavers entering tertiary education. Methods. A cross-sectional survey among first-year students, aged 18-25 years, registered at one of three universities chosen for the study in Johannesburg, South Africa. Informed consent was obtained prior to completing a self-administered, close-ended, structured questionnaire. Factors associated with poor knowledge or high risk perception were identified using modified Poisson regression.

Results. In total, 792 students were included; 53.3\% $(n=438)$ were categorised as having poor TB knowledge and 52.1\% $(n=412)$ poor HIV knowledge, while $43.4 \%(n=344)$ were categorised as having high TB risk perception and $39.8 \%(n=315)$ high HIV risk perception. Male students were more likely to have poor knowledge of HIV and perceive themselves at risk of acquiring HIV. Low socioeconomic status was associated with a high risk perception of HIV. One in 3 participants (30.6\%) stated that they had never had an HIV test. In total, 24 students (9 male, 15 female) reported that they were HIV-positive, of whom 15 (62.5\%) were on antiretroviral therapy. Only $14.1 \%$ had been screened for TB in the past 6 months.

Conclusion. The findings indicate a need to enhance health promotion activities among university students so as to aid preventive strategies for reducing the burden of HIV and TB infection.
\end{abstract}

S Afr J Child Health 2018;12(2 Suppl 1):S19-S31. DOI:10.7196/SAJCH.2018.v12i2.1525

South Africa (SA), with an estimated incidence of 781 cases of TB per 100000 population in 2016, is one of the countries with the highest burden of the disease, and it continues to be a major health problem in the country. ${ }^{[1,2]}$ The relationship between TB and HIV is well documented, and HIV is a major contributor to the TB epidemic. The risk of developing TB among HIV-positive individuals is estimated to be 16-27 times higher than among those who are HIV negative. ${ }^{[3]}$ HIV primarily affects people in their most productive years and, in SA, young people (ages 15 - 24) have been identified as a high-risk group. ${ }^{[4]}$

Adolescents and young adults face difficult and often confusing emotional and social pressures as they move from childhood to adulthood. Compared with adults, young people generally lack sufficient knowledge about HIV and are less likely to be tested. They also need more support to navigate healthcare and access services. ${ }^{[-7]}$ The barriers and facilitators of HIV counselling and testing (HCT) among young people have been well described. ${ }^{[7-9]}$ Potential barriers include stigma, discrimination, concerns about confidentiality, lack of adequate housing, education, employment and psychosocial support. Poor HIV and sexual health knowledge, among young women in particular, has been identified as a barrier to HIV testing and prevention. ${ }^{[7-9]}$ Studies have shown that young people's behaviour and how they perceive their risk of acquiring HIV can influence their intentions or decisions to test (e.g. individuals engaging in unsafe sexual practices suspect a positive result and are less likely to test). ${ }^{[10-12]}$

It is unclear if adolescents and young adults leaving high school and entering tertiary education are adequately equipped for the social complexities such as peer pressure, complex social networks or unequal power dynamics in sexual decision-making that they may encounter in their new environment. It is also unclear whether they can make informed decisions about their sexual behaviour so as to protect themselves and others from becoming infected with HIV. In 2000, SA implemented the HIV and AIDS Life Skills Programme in all primary and secondary schools. HIV education, under the Integrated School Health Programme (ISHP), aimed to make youth-friendly, sexual and reproductive health services accessible in schools with the intention of supporting HIV prevention efforts. Changes to the policy later included HCT in the range of services offered to high school learners. ${ }^{[7]}$ Between 2013 and 2014, the proportion of schools implementing the ISHP dropped significantly from $60 \%$ to $20 \%$, respectively. ${ }^{[4,13]}$ While benefits of the ISHP have been described in the high school setting, ${ }^{[7]}$ it is not clear if young people understand the importance of HCT or if they access HCT services once they have left high school in response to changes in their behaviour and/or risk perception. 
According to the South African National Department of Higher Education and Training, over one million students enrolled in universities in South Africa for the 2016 academic year, of whom $20 \%$ were first-time students. As there is an increasingly diverse body of students entering university in South Africa, the university campus presents an important opportunity to assess students' knowledge, risk perception and health-seeking behaviour regarding TB and HIV, and identify opportunities to intervene with health promotion activities. The present study adds to the limited, but growing, body of literature that looks at the interaction between the factors and behaviour of young people entering tertiary education, while informing policy on the development of youth-friendly health services and support. ${ }^{[7]}$ The study aims to describe knowledge and risk perception of TB, HIV and access to healthcare services among high school leavers entering tertiary education.

\section{Methods \\ Study design}

This was a cross-sectional survey among first-year students, aged 18 - 25 years, registered at one of three tertiary institutions (universities) chosen for the study in Johannesburg, South Africa.

\section{Setting and population}

Gauteng comprises the largest share of the South African population, with $\sim 13.5$ million people living in the province. Johannesburg has a population of 4.5 million, making it the largest South African city and one of the largest African cities. Johannesburg has an overall TB incidence of 326 cases per 100000 population. ${ }^{[5]}$ The three universities selected for this study are located in Johannesburg, with less than $40 \mathrm{~km}$ between them. The universities, with a total annual intake of 19000 first-year students, offer a diverse range of undergraduate programmes.

The study population included all first-year students aged 18-25 years (students could thus sign written informed consent without parent/legal guardian permission) who were registered between February 2017 and November 2017 at one of three tertiary institutions (universities) chosen for the study in Johannesburg (two government subsidised and one private university). A convenience sample was obtained by approaching first-year students in common areas on the days that study staff (i.e. interviewers) visited the university campuses (e.g. library, canteen, lunch area). Because the target population of the study was high school leavers entering tertiary education, students who had completed secondary school more than three years ago and those who had been university students for more than one year (e.g. those completing a bridging year prior to registering for a formal degree) were excluded.

\section{Study procedure}

Study staff approached potential participants and those who met the initial pre-screening criteria (e.g. first-year student and registered at the university) were invited to participate. Thereafter, study staff provided a detailed explanation of the study and confirmed eligibility, and eligible students were asked to provide written informed consent. Students enrolled in the study completed a self-administered, close-ended structured questionnaire. Both the informed consent and questionnaire were available in English only. The questionnaire contained questions on participant demographics, HIV knowledge, HIV risk perception, TB knowledge and TB risk perception. Questions relating to knowledge and risk perception were derived from published questionnaires ${ }^{[14-21]}$ and adapted for the local context. Assessment of knowledge (36 items for TB and 42 items for HIV) included questions about cause, mode of transmission, symptoms, risk factors, prevention, treatment and where to obtain help if sick, ways of preventing disease, and treatment for HIV and TB. Questions on risk perception (10 for TB and 20 for HIV) included questions that reflected common myths and misconceptions and also focused on perceived susceptibility to TB/HIV, perceived benefits and barriers to seeking care and disclosure of TB and/or HIV Questions on HIV risk perception included additional questions from the validated perceived risk measure as reported by Napper and colleagues. ${ }^{[22]}$ Study staff helped the participants to complete the self-administered paper-based questionnaire and then entered the responses into REDCap, an electronic data entry tool, hosted at the University of the Witwatersrand. ${ }^{[23]}$

\section{Sample size and weighting}

OpenEpi epidemiological calculator for prevalence studies was used to calculate the sample size (http://www.openepi.com/SampleSize/ SSPropor.htm). Using an estimated population of 19000 firstyear university students across the three universities (regardless of gender), an anticipated percentage frequency of $50 \%$, and a confidence limit of $\pm 5 \%$, the estimated sample size calculated to have sufficient power to detect true level of knowledge was 634 . Taking into account a $20 \%$ non-response rate, the total sample size increased to 792. We further weighted the sampling according to the estimated number of first-year vacancies that each university had, so that participants were enrolled at a ratio of 3:6:1 for each university. During the study period, study staff (i.e. interviewers) rotated through the 3 universities and 6 campuses to enrol 792 firstyear students.

\section{Study variables}

Socioeconomic status (SES) was measured using an asset index, based on ownership of assets, power source, and food security as recommended by Filmer and Pritchett (1998). ${ }^{[24]}$ Assets were combined into a wealth index using weights derived through principal component analysis (PCA). The PCA involves breaking down assets (e.g. type of dwelling, radio, refrigerator) or household service access (e.g. electricity, access to water, sanitation etc.) into categorical or interval variables. The variables were then processed to obtain weights and principal components. Based on this index, SES of households was divided into three categories (low, medium and high) representing proxies for SES.

The high school where participants obtained their senior certificate after Grade 12 schooling was recoded as either a private or public high school according to name of school and city or province where the school was located. In most instances, this involved accessing the school's public website to ascertain this. Race or ethnicity was classified as reported by participants (i.e. self-identifying).

\section{Outcomes, data and data analysis}

The primary outcomes of the study were the proportion of students with poor knowledge of HIV or TB and a high risk perception of HIV or TB.

We assigned a number to participants' responses so that a score could be calculated and categorised. For TB and HIV knowledge, fourpoint Likert items ('True,' 'Probably True', 'Probably False' and 'False') measuring either a positive or negative response to a statement were summed to create a score for the group of items. Questions with 'Yes' and 'No' response options were recoded and added to the score (e.g. a true response to the question was given 2 points and a false response 0 ). The total score was then split into higher and lower knowledge level based on the median score (i.e. less than the median as low/ poor knowledge, and more than or equal to the median as high/ adequate knowledge). For TB and HIV risk perceptions, a similar approach was taken. Using four-point Likert items, participants indicated their agreement with the statement ('Strongly agree,' 'Agree', 'Disagree' and 'Strongly Disagree'). Questions for HIV risk perception that had other response options were recoded and added to the score. ${ }^{[14]}$ The total score was split into low and high risk perception 
based on the median score. Missing or 'Refused to answer' responses were not included in the data coding, whereas 'Don't know' was regarded as a negative response to the statement and scored accordingly. Internal consistency of each set of questions was calculated using Cronbach's method, and the alpha coefficient presented. In addition, we report the completeness of data and the average number of data fields missing for each outcome.

Participant demographics (at enrolment) are presented using proportions for categorical variables and medians with corresponding interquartile ranges (IQRs) for continuous variables, and stratified by university. Continuous data were compared using the Kruskal-Wallis for non-parametric or $t$-test for parametric data, where appropriate, while the chi-square test (or Fischer Exact test for sparse data) was used to compare proportions.

We used modified Poisson regression to estimate the association between student characteristics (e.g. gender, nationality, SES etc.) and our primary outcomes. We present the crude or univariate estimate with the 95\% confidence interval for each factor. Factors with $p<0.1$ in the univariate model along with other potential confounders $(10 \%$ difference between the crude and adjusted estimates) and a priori variables (e.g. age, gender, university, SES) were included in the final multivariate model. To minimise issues with highly correlated variables, we used principal component analysis (PCA) - a method that combines the variables in a non-correlated way - to create a new variable (e.g. SES) which was included in the model. All analyses were conducted using SAS version 9.3 (SAS Institute, USA).

The present study was approved by the Human Research Ethics Committee (Medical) of the University of the Witwatersrand (Certificate number M161019). All participants provided written informed consent to participate in the study.

\section{Results}

A total of 1656 students were approached to participate in the study. A third of the students $(32.1 \%, n=532)$ approached did not have time to participate in the study, $5 \%$ $(n=84)$ were not interested in participating and $7.8 \% \quad(n=130)$ were not university students or first-year students. Of those interested in participating and screened $(n=910), 811$ were eligible to participate and were enrolled. Of these, 792 were included in the analysis after fictitious data $(n=5$; where students fabricated data), duplicates $(n=11)$ and those with incomplete consent $(n=3)$ had been removed (Fig. 1). The age and gender of those included in the analysis were representative of all students approached, screened and enrolled (mean age 19.4 v. 19.2, 18.0 and 19.3 years; male $44.4 \%$ v. $40.9 \%$, $43.5 \%$ and $44.6 \%$ ).

The number of participants enrolled at each university reflected the relative size of the university population (i.e. $n=228$, $28.8 \% ; n=480,60.6 \%$ and $n=84,10.6 \%)$. The majority of students were of the Christian religion (88\%), single (98.6\%) and studying full-time (98.7\%), with only a few students (5.5\%) reporting that they were employed. Participants were predominantly between the ages of 19 and 25 years (65.8\%), of black ethnicity (91.2\%), South African (73.7\%), female (54.8\%), and mostly public high school graduates $(74.4 \%)$ who completed high school in Gauteng Province (59.6\%) (Table 1). Compared with participants registered at the two government-subsidised

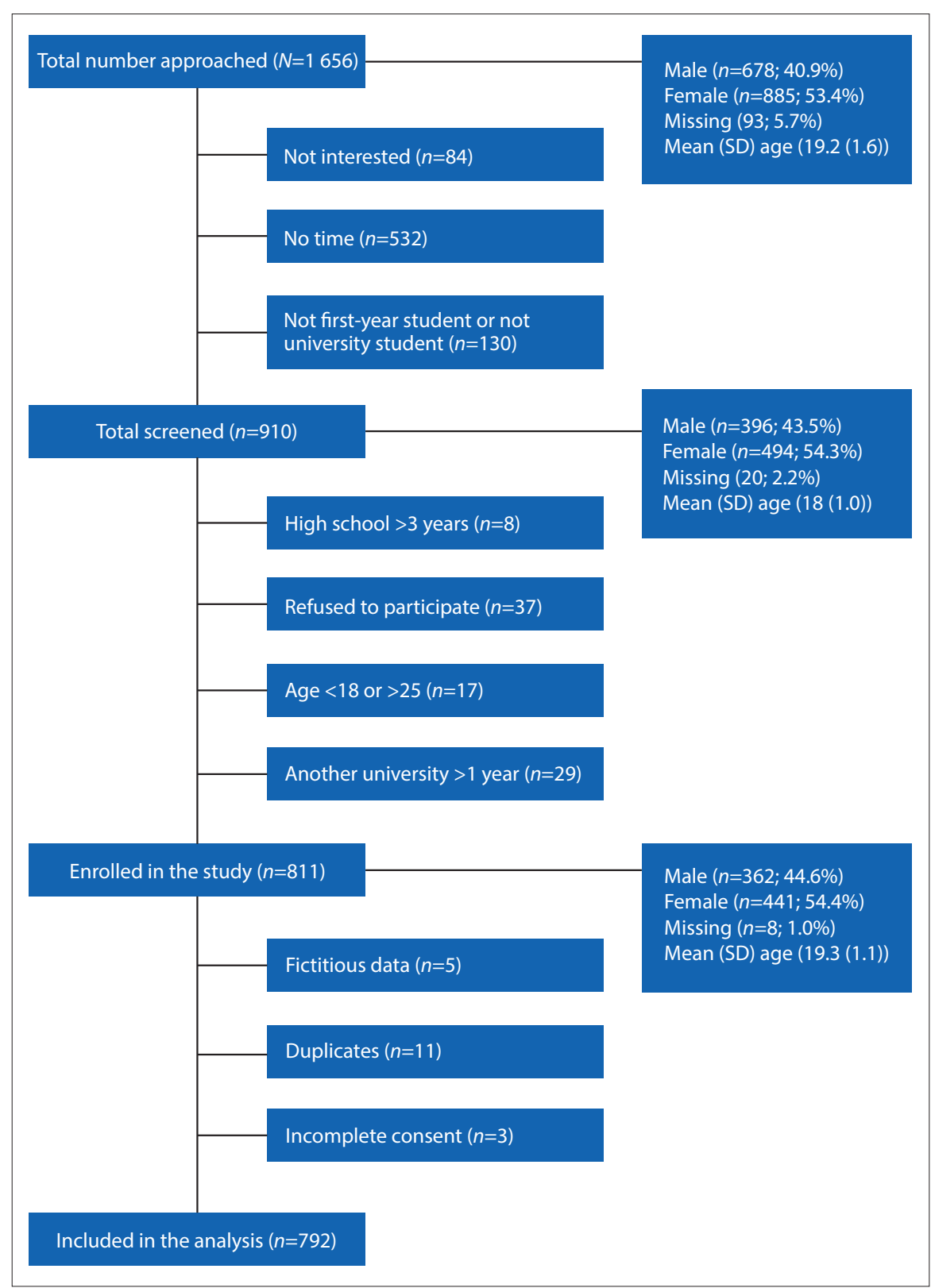

Fig. 1. Summary of study enrollment process. ( $S D=$ standard deviation.) universities, the private university $(n=84)$ had more female $(61.9 \%$ v. $57.0 \%$ and $52.5 \%)$ and more white students $(13.1 \%$ v. $4.4 \%$ and $1.3 \%$ ) and a higher SES as measured by higher-than-average monthly household income, attended a private high school, tuition paid by parents, living with spouse/ partner/parent, private health insurance and high SES according to the PCA $(p<0.05)$.

\section{Knowledge and risk perception}

Prior to the analysis, we tested the internal consistency and completeness of the data, as presented by each outcome (Table 2). had the lowest internal consistency $(<0.50$ indicates that the items are not appropriate; $\geq 0.70$ is preferred). In particular, students appeared to struggle with the question 'I live Questions related to TB risk perception 


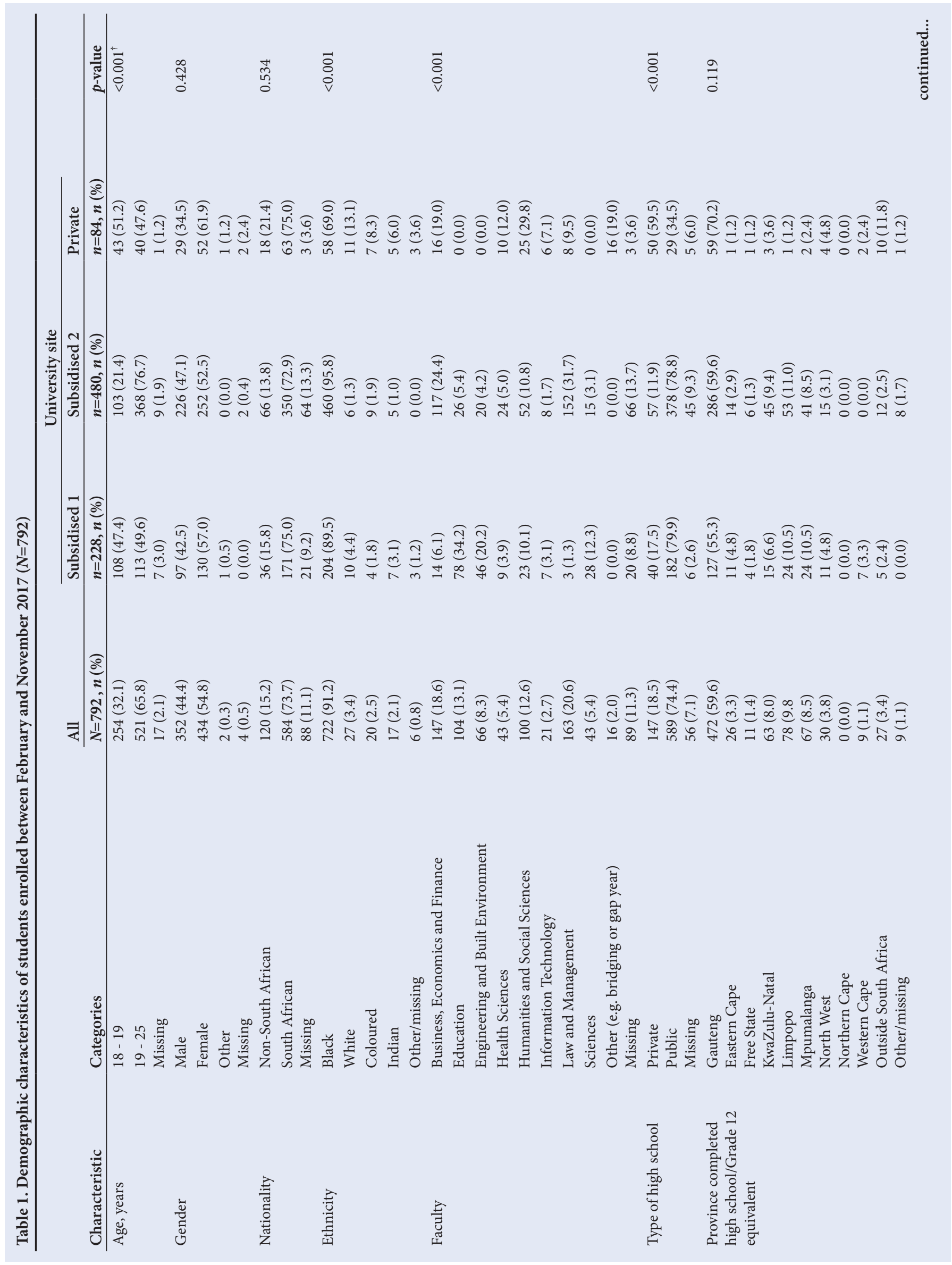




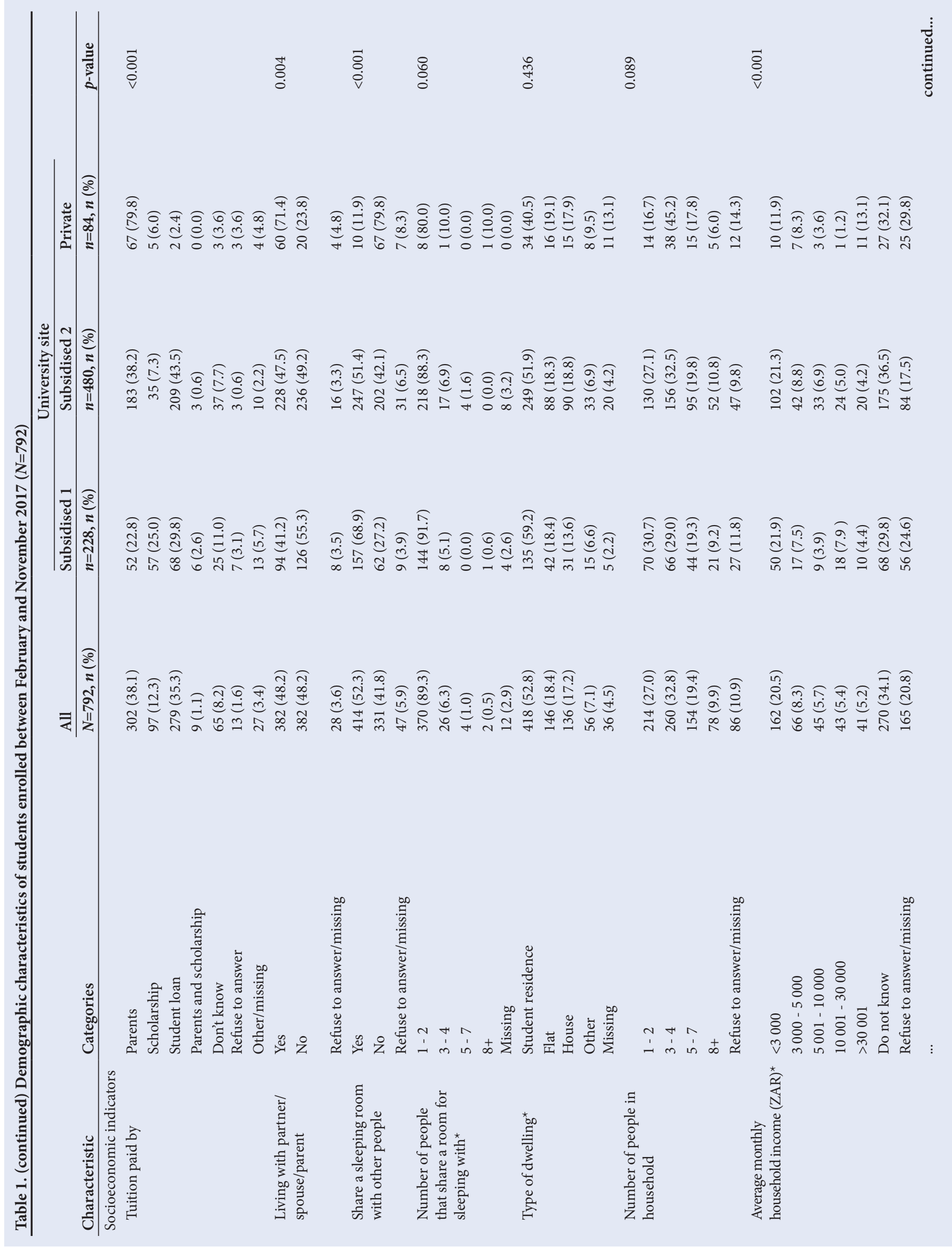




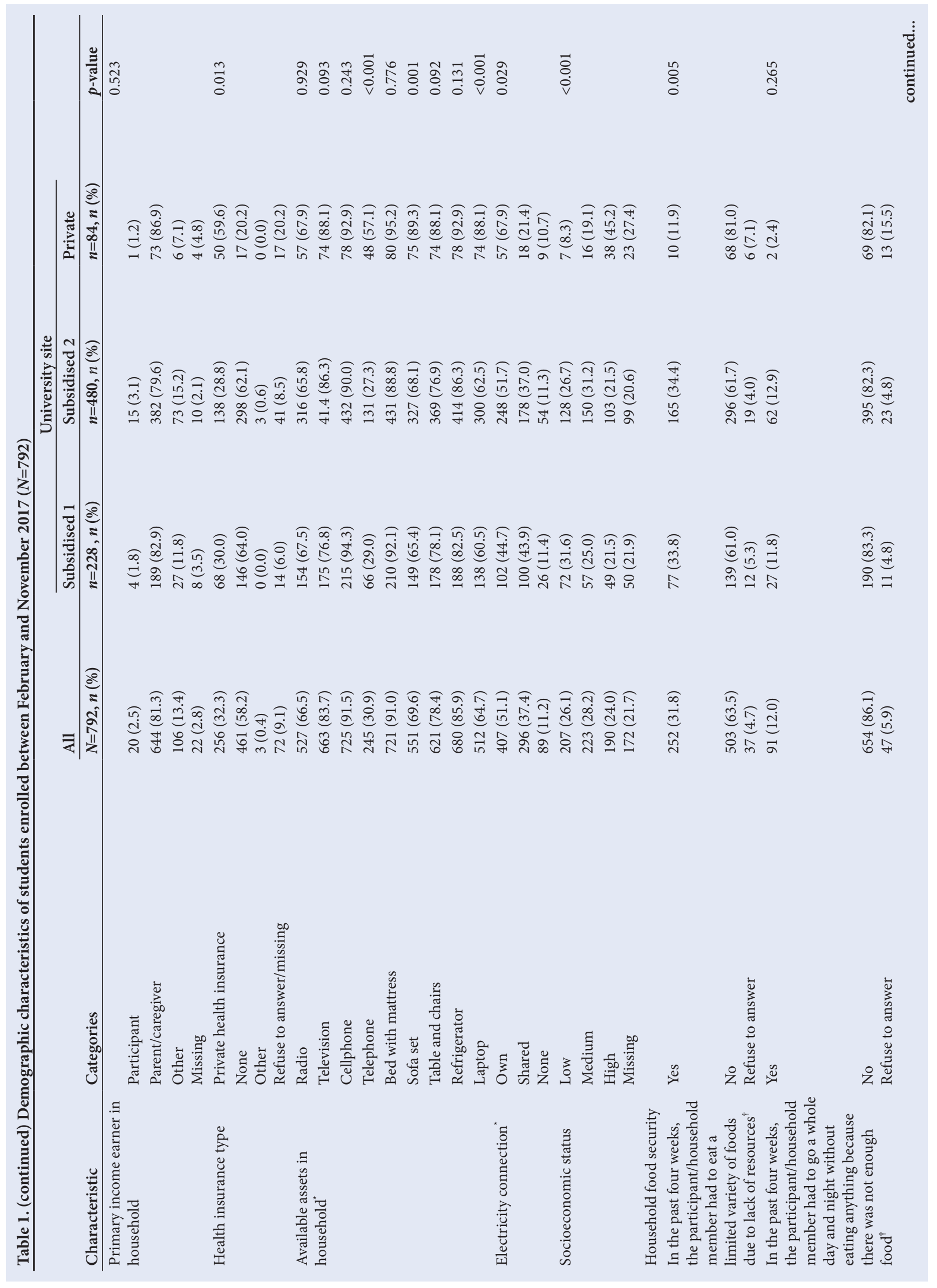




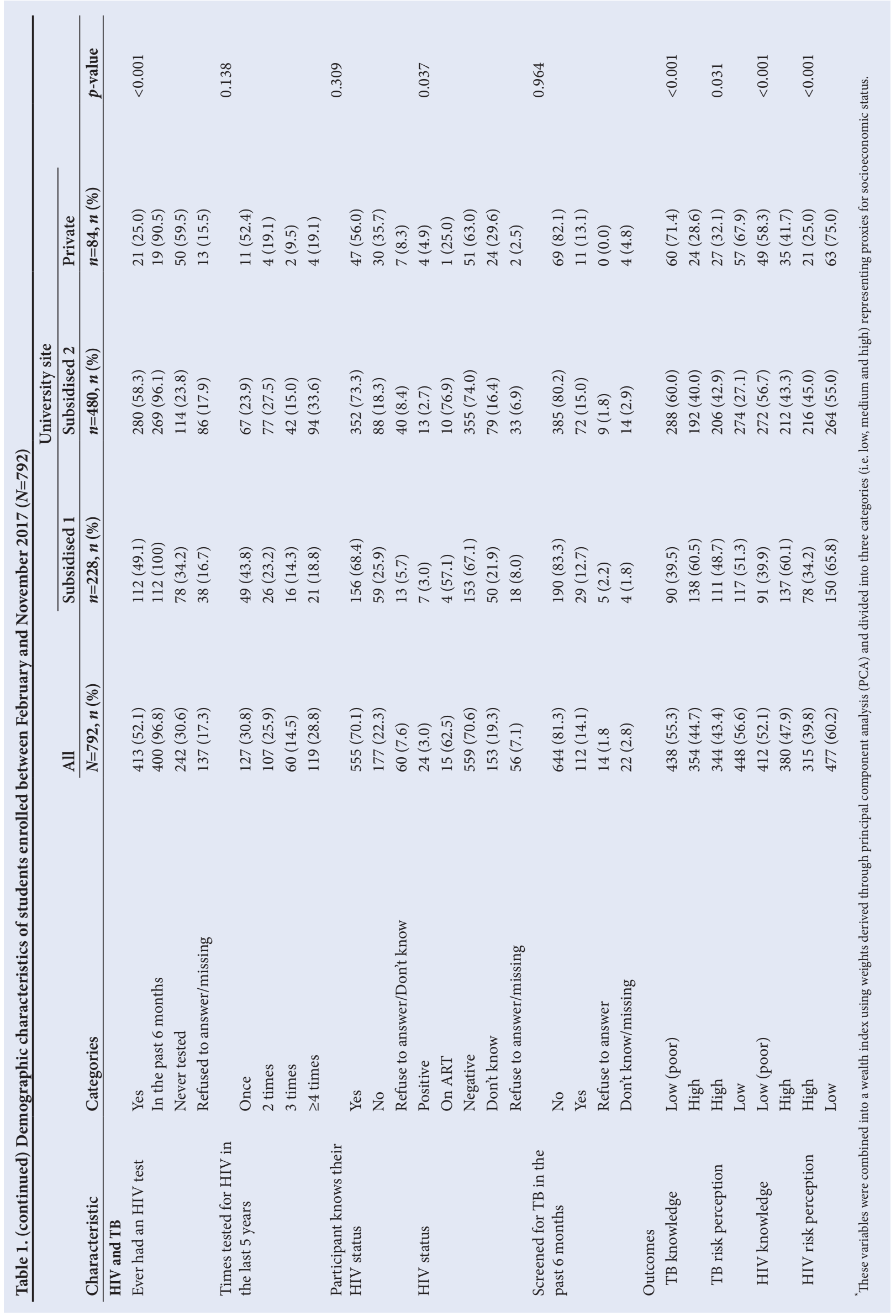


Table 2. Summary of the internal consistency and completeness of questionnaire data

\begin{tabular}{|c|c|c|c|}
\hline Questionnaire data & Items & Cronbach's alpha & $\begin{array}{l}\text { Missing (per item), } \\
\text { median (IQR); min. - max. }\end{array}$ \\
\hline TB knowledge & 36 & 0.77 & $10(7-15) ; 0-30$ \\
\hline HIV knowledge & 42 & 0.74 & $9.5(8-13) ; 0-44$ \\
\hline TB risk perception & 10 & 0.50 & $18(15-26) ; 11-96$ \\
\hline HIV risk perception & 20 & 0.60 & $26(17-35) ; 11-69$ \\
\hline
\end{tabular}

together with a lot of people in a crowded place so could get TB.' which had the highest non-response rate $(12 \%, n=96)$.

Based on our definition, 55.3\% $(n=438)$ and $52.1 \%(n=412)$ were categorised as having poor TB or HIV knowledge while $43.4 \%$ $(n=344)$ and $39.8 \%(n=315)$ were categorised as having high TB or HIV risk perception. Compared with participants registered at the two government-subsidised universities, those registered at the private university $(n=84)$ had poorer knowledge of TB and HIV and perceived their risk of acquiring TB or HIV as low $(p<0.05)$.

Compared with female participants, male students were more likely to have poor knowledge of HIV (relative risk (RR) 1.21, 95\% confidence interval (CI) 1.04-1.46) and perceive themselves at risk of acquiring HIV (RR 1.63, 95\% CI 1.25 - 2.12) (Table 3). Compared with those with a high SES, individuals with a low SES perceived themselves at risk of acquiring HIV (high v. low SES RR 0.67, 95\% CI $0.46-0.98 ; p<0.05)$. Other factors, such as no health insurance, tuition paid by loans or scholarships, or not living with spouse/ partner/parent, supported this finding ( $p<0.05$ in the univariate) and contributed to a high TB and HIV risk perception (Tables 3 and 4).

\section{HIV and TB health-seeking behaviour}

One in 3 students $(n=242 ; 30.6 \%)$ reported that they had never had an HIV test, and this figure was significantly higher (59.5\%) among students attending the private university. Of the students who reported knowing their HIV status $(n=555), 44 \%$ reported that they had never had an HIV test, which suggests that many students assume their status despite having never been tested, perhaps because they had not been sexually active or considered themselves at low risk. ${ }^{[7]}$ Among those who had had an HIV test $(n=413 ; 52.1 \%)$, the majority had been tested in the previous 6 months and, for a third $(n=127 ; 30.8 \%)$ of them, this was the only test reported within the last 5 years, possibly as a result of active participation in HIV wellness days arranged by the universities. In total, 24 participants $(9$ male and 15 female) reported that they were HIV-positive and, of these, $15(62.5 \%)$ were on antiretroviral therapy. Less than $15 \%$ of students enrolled (14.1\%) had been screened for TB in the past 6 months. HIV testing and TB screening behaviour was similar among male and female participants $(p>0.05)$.

\section{Discussion}

Compared with adults, young people generally lack sufficient knowledge about HIV and are less likely to be tested. ${ }^{[9]}$ We show that, compared with females, males have a poorer knowledge of TB $(62.5 \%$ v. $49.3 \%$ ) and HIV (56.8\% v. $47.0 \%$ ), which is contradictory to other reports ${ }^{[25,26]}$ but might be because adolescent women are more likely to engage in healthcare services (i.e. contraceptive services or antenatal care, which serve as potential sources of information) in our setting. The present study shows that low SES was associated with a high-risk perception of HIV. The disproportionate burden of HIV disease and HIV fear among the poor and vulnerable in South Africa has been described ${ }^{[27]}$ Those with a poor SES report lower frequency of HIV testing, poorer access to HIV information, more stigmatising attitude towards HIV, and high personal HIV risk perception. ${ }^{[27]}$ Exposure to social media and interpersonal communication may be responsible for the lower HIV risk perception observed among those with a high SES. That many of the students had access to radio, television and computers/laptops, in particular those attending the private university, may explain this finding. Furthermore, gender differences seem to exist in perceptions of the risk of acquiring HIV, with males having a higher risk perception than females.

Results from the present study show that the prevalence of HIV among university students in Johannesburg, who reported their HIV status, was slightly lower than what has been reported nationally among young adults (15 - 24 years) $(4.1 \%$ v. $4.6 \%){ }^{[28]}$ As only $63.1 \%$ of those who responded admitted to ever having an HIV test, this number is likely to be an underestimate of HIV prevalence in this population. Less than $15 \%$ of participants had been screened for TB in the past 6 months. Because of high rates of TB/HIV co-infection, TB screening and services could serve as the entry point for HIV testing or facilitate the link to HIV counselling and testing (HCT), as a gateway to treatment and prevention services.

Adolescents and young adults are at increased risk for HIV and are the one group worldwide where reduction in new HIV cases or HIV-related mortality has not been observed. ${ }^{[29]}$ Because adolescents are at high risk for both acquiring and transmitting HIV, they should be considered a priority in the development of HIV, TB and STI prevention strategies. ${ }^{[29]}$ Addressing the health needs of this unique population is critical in order to achieve the UNAIDS 90:90:90 targets. ${ }^{[30]}$ In particular, strategies to improve HCT as an entry point into the treatment and prevention cascade, including prevention, clinical management and psychosocial support, will be important. ${ }^{[5,29]}$ Two core groups should be considered when developing or testing new technologies or interventions for this group: HIV-negatives with a focus on prevention and the reduction of new infections, and HIV-positives with a focus on testing, treatment and ultimately viral load suppression.

\section{Study limitations}

The results of this study should be considered in the light of the study limitations. Firstly, we included 3 out of more than 30 universities across SA, which may limit the generalisability of the study findings. To minimise selection bias, we included 1 private and 2 of the biggest public universities in SA - one a traditional university which offers theoretically oriented degrees and the other a comprehensive university which offers both theoretically oriented and vocationally oriented diplomas and degrees. Secondly, we only enrolled students available during study visits to the campus, which limited our ability to enrol part-time students, those attending night classes and those registered for distance education. Thirdly, Cronbach's alpha, which is considered to be a measure of scale reliability or strength of consistency was low for TB risk perception (0.50), so the results should be interpreted with caution. The use of Likert scales may introduce bias which may include central tendency bias (where participants avoid using extreme response categories), acquiescence 


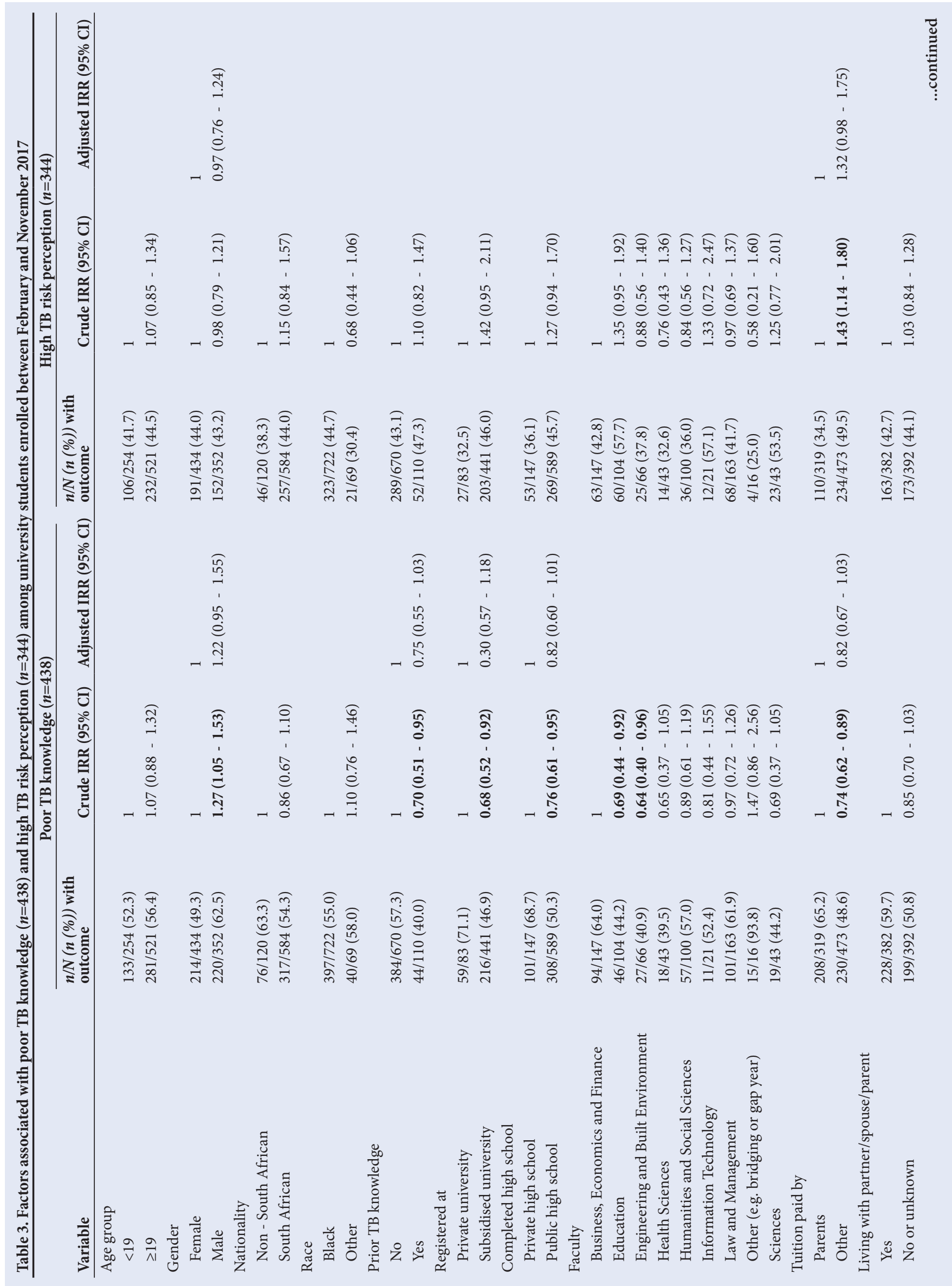




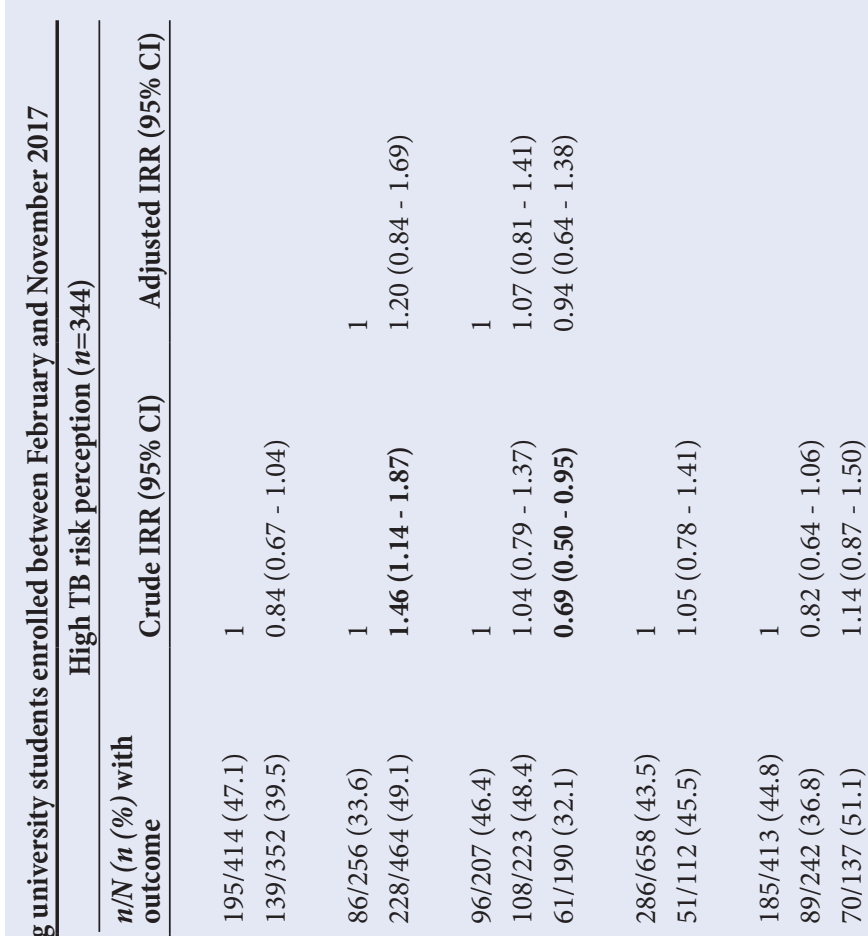

$+$
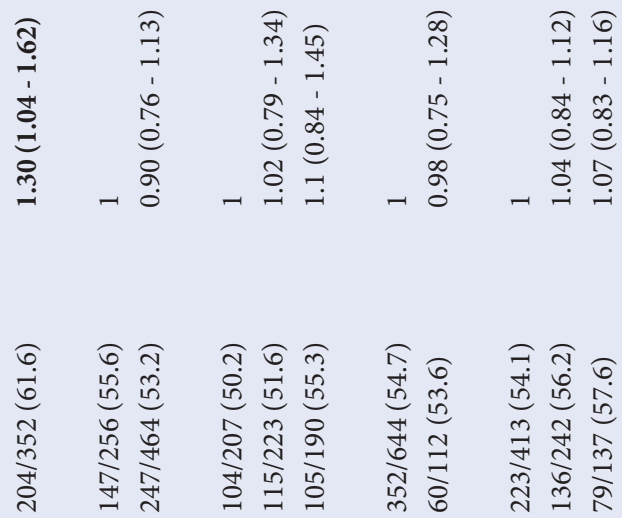
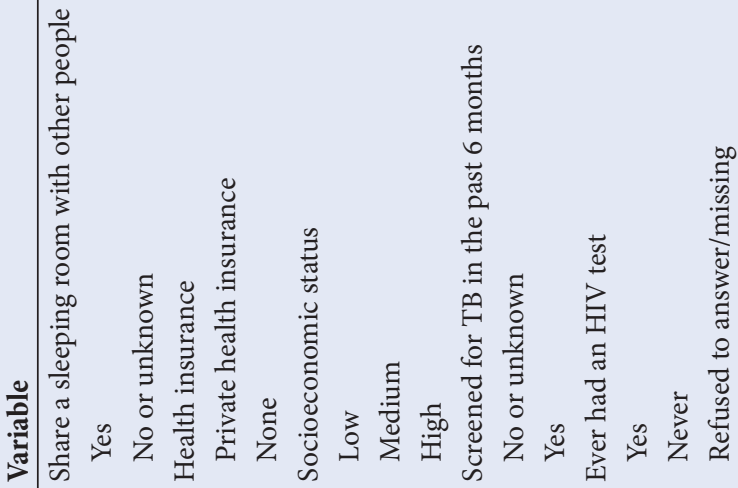

bias (where participants agree with the statement presented) or social desirability bias - presenting themselves in the best possible light. The questionnaires that were used contained an equal number of positive and negative statements, which helped to alleviate the problem of acquiescence. However, central tendency and social desirability were more difficult to overcome. The questionnaire was also self-administered by participants, which minimised the introduction of interviewer bias but in some instances might have resulted in more missing data. Missing data also compromised the ability to calculate the PCA and derive SES for $21.7 \%$ of the participants. For almost half of the participants, the SES was that of the student whereas for others (those living with parents/guardians) the SES reflects that of their parents/guardians. Lastly, there was subjective reporting of participant data of TB/HIV testing and test results, which only relied on participants' recall ability.

\section{Conclusion}

Adolescents and young adults leaving high school and entering tertiary education not only lack sufficient knowledge, but also perceive themselves at risk of acquiring HIV and/or TB. Knowledge and risk perception differ by gender and SES, where males and those with low SES had poorer knowledge of HIV and perceived themselves at risk of acquiring HIV. Participants attending two public universities had poorer knowledge and higher risk perception than those at a private university. Despite organised wellness days for HCT and access to healthcare services on campus, many participants did not know their HIV status and had never been tested for HIV. The present study, while demonstrating gaps in knowledge about TB and HIV, highlights the need to enhance health promotion activities among university students and provide additional support to improve testing behaviour. The university campus offers an opportunity to intervene and perhaps change the way that we reach and engage adolescent men in HCT.

Disclaimer. This study was made possible by the generous support of the people of the USA through Cooperative Agreement AID 674-A-12-00029 of the United States Agency for International Development (USAID). The contents of the article are the responsibility of the authors and do not necessarily reflect the views of USAID or the United States Government. The funders had no role in the study design, collection, analysis and interpretation of the data, and in the manuscript preparation or the decision to publish.

Acknowledgements. The authors wish to thank the staff and students at the universities who supported and participated in this study. A special Thank You to Alice Kono, Busi Sithole, Melda Musina, Portia Ngwenya, Vinolia Ntjikelane, Barbara Xhosa and Given Malete for all their help with data collection, quality assurance and data management. The support of the DST-NRF Centre of Excellence in Human Development towards this research is hereby acknowledged. Opinions expressed and conclusions arrived at, are those of the authors and are not necessarily to be attributed to the $\mathrm{CoE}$ in Human Development. The authors gratefully acknowledge the support of the Demography and Population Studies Programme, Schools of Public Health and Social Sciences, Faculties of Health Sciences and Humanities, University of the Witwatersrand, Johannesburg, South Africa.

Author contributions. DE, EL, PN and LL were involved in the study conception and design. DE and NM were involved in 


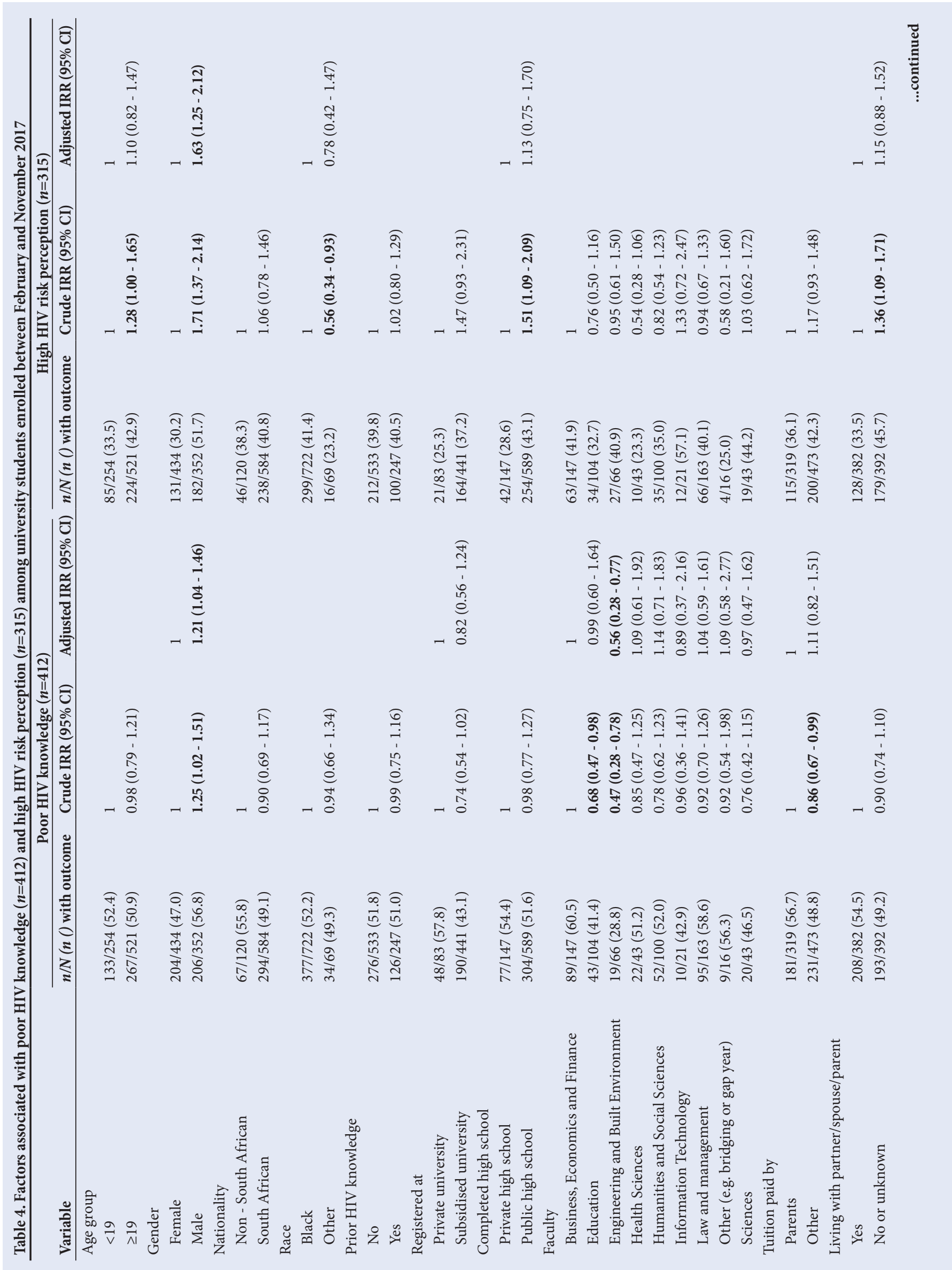




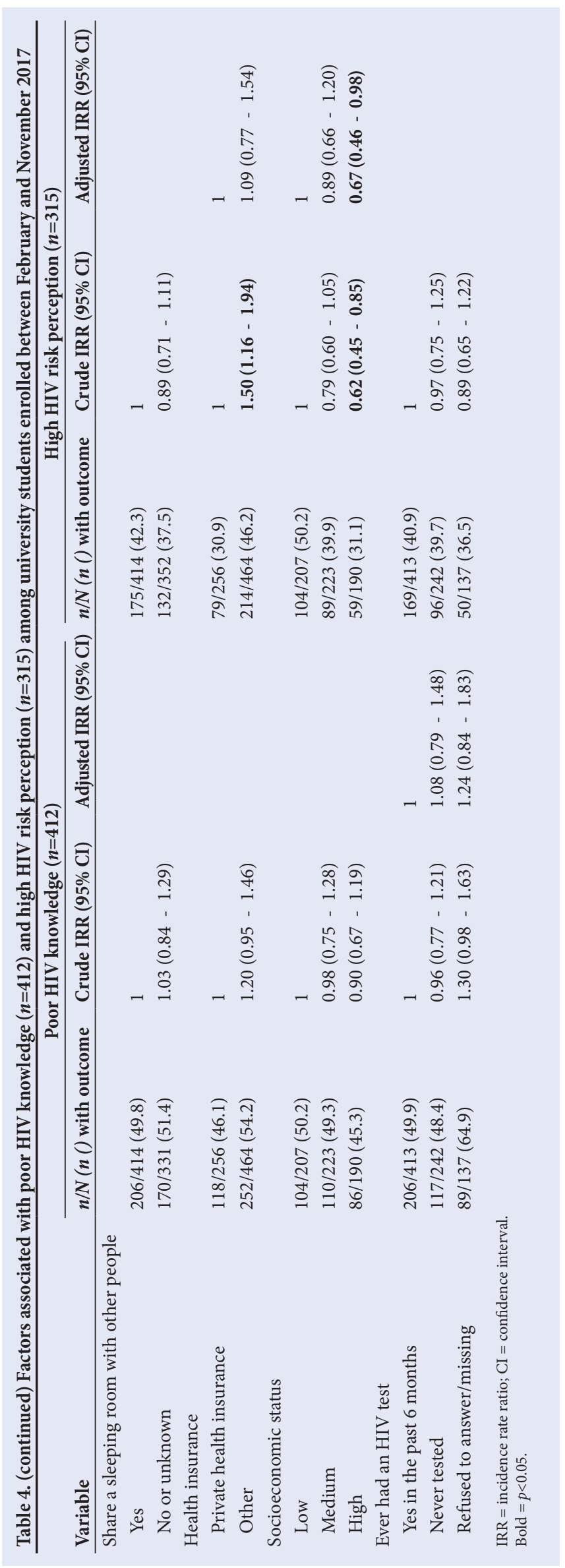

project administration and study implementation while $\mathrm{DE}, \mathrm{NM}$ and $\mathrm{CN}$ undertook data management. SA, CL and NM were involved in developing data collection tools, data collection and verification. EL and DE were the supervisor and co-supervisor of SD's Master of Medicine research project (CL and SA). DE, NM, CN and PN were involved in data analysis and interpretation of data. LL, EL, JB and PN were involved in interpretation of the results and contributed to the Discussion and Limitations sections. DE drafted the first manuscript and all authors were involved in editing, revising and critically reviewing it for important intellectual content and final approval of the manuscript. LL provided resources and funding for the project.

Funding. DE, CN, NM and LL were supported through the South Africa Mission of the United States Agency for International Development (USAID) under the terms of Cooperative Agreement USAID674-A-12-00029 of the Health Economics and Epidemiology Research Office.

\section{Conflict of interests. None.}

1. Mlotshwa M, Abraham N, Beery M, et al. Risk factors for tuberculosis smear non-conversion in Eden district, Western Cape, South Africa, 2007 2013: A retrospective cohort study. BMC Infect Dis 2016;16:365. https://doi. org/10.1186/s12879-016-1712-y

2. Statistics South Africa. Mortality and causes of death in South Africa, 2014: Findings from Death Notification. Pretoria: StatsSA; 2015.

3. Corbett E, Watt C, Walker $\mathrm{N}$, et al. The growing burden of tuberculosis: Global trends and interactions with the HIV epidemic. Arch Intern Med 2003;163:1009-1021.

4. Shisana O, Rhele T, Simbayi L, et al. South African National HIV Prevalence, Incidence and Behaviour Survey, 2012. Cape Town: HSRC Press; 2014.

5. Lawrence E, Struthers P, Van Hove G. HIV counselling and testing in secondary schools: What students want. S Afr J HIV Med 2015;16(1):390.

6. Maughan-Brown B, Lloyd N, Bor J, Venkataramani A. Increasing access to HIV testing: Impacts on equity of coverage and uptake from a national campaign in South Africa. Southern Africa Labour and Development Research Unit Working Paper Number 145. Cape Town: SALDRU, University of Cape Town; 2015.

7. Strauss M, Rhodes B, George G. A qualitative analysis of the barriers and facilitators of HIV counselling and testing perceived by adolescents in South Africa. BMC Health Services Research 2015;15:250.

8. UNAIDS. Prevention Gap Report. Geneva: UNAIDS, 2016. http://www. unaids.org/sites/default/files/media_asset/2016-prevention-gap-report_en.pdf (accessed 28 December 2017).

9. Dawood H. Adolescent HIV treatment issues in South Africa. S Afr Med J 2015;105(11):1-3.

10. Ikechebelu IJ, Udigwe GO, Ikechebelu N, Imoh LC. The knowledge, attitude and practice of voluntary counselling and testing (VCT) for HIV/AIDS among undergraduates in a polytechnic in Southeast, Nigeria. Niger J Med 2006;15(3):245-249.

11. Njagi F, Maharaj P. Access to voluntary counselling and testing services: Perspectives of young people. S Afr Rev Sociol 2006;37(2):113-127.

12. Fako TT. Social and psychological factors associated with willingness to test for HIV infection among young people in Botswana. AIDS Care 2006;18(3):201-207.

13. African National AIDS Council. Global AIDS Response Progress Report Pretoria: SANAC; 2015. http://sanac.org.za/wp-content/uploads/2016/06/ GARPR_report-high-res-for-print-June-15-2016.pdf (accessed 28 December 2017).

14. Chizimba R, Christofides N, Chirwa T, et al. The association between multiple sources of information and risk perceptions of tuberculosis, Ntcheu district, Malawi. PLoS One 2015;10(4):e0122998. https://doi.org/10.1371/journal. pone. 0122998

15. Nyasulu P, Phiri F, Sikwese S, et al. Factors influencing delayed health care seeking among pulmonary tuberculosis suspects in rural communities in Ntcheu District, Malawi. Qual Health Res 2016;26:1275-1288. https://doi. org/10.1177/1049732315588083

16. Devine M. TB Awareness Survey TB North East London Project. Health Works in London, 2006. file://C:/Users/denisee/Downloads/TB\%20Awareness\%20 Survey\%20Report\%202006\%20(1).pdf (accessed 28 December 2017).

17. Cavric G. Knowledge of HIV/AIDS, related attitudes and participation in risky sexual behaviour among first and fourth year female students at the University of Botswana. 2011. http://wiredspace.wits.ac.za/bitstream/ handle/10539/11328/G.\%20Cavric\%20MPH\%20Theses\%2028\%20March\%20 2011.pdf? sequence $=1$ \&isAllowed $=y$ (accessed 28 December 2017).

18. Fatiregun AA, Ejeckam CC. Determinants of patient delay in seeking treatment among pulmonary tuberculosis cases in a government specialist hospital in Ibadan, Nigeria. Tanzan J Health Res 2010;12:113-120. https://www.ajol.info/ index.php/thrb/article/viewFile/56398/44833 
19. Maneze D, DiGiacomo M, Salamonson Y, Descallar J, Davidson PM. Facilitators and barriers to health-seeking behaviours among Filipino migrants: Inductive analysis to inform health promotion. BioMed Res Int 2015:506269. https://doi. org/doi:10.1155/2015/506269

20. Fox MP, Mazimba A, Seidenberg P, Crooks D, Sikateyo B, Rosen S. Barriers to initiation of antiretroviral treatment in rural and urban areas of Zambia: A crosssectional study of cost, stigma, and perceptions about ART. J Int AIDS Soc 2010; 13:8. https://doi.org/10.1186/1758-2652-13-8

21. Wingfield T, Boccia D, Tovar M, et al. Defining catastrophic costs and comparing their importance for adverse tuberculosis outcome with multi-drug resistance: A prospective cohort study, Peru. PLoS Medicine 2014;11(7):e1001675. https://doi. org/10.1371/journal.pmed.1001675

22. Napper LE, Fisher DG, Reynolds GL. Development of the perceived risk of HIV scale. AIDS Behav 2012;16:1075-1083. https://doi.org/10.1007/s10461011-0003-2

23. Harris PA, Taylor R, Thielke R, Payne J, Gonzalez N, Conde JG. Research electronic data capture (REDCap) - a metadata-driven methodology and workflow process for providing translational research informatics support. J Biomed Inform 2009;42(2):377-381. https://doi.org/10.1016/j.jbi.2008.08.010

24. Filmer D, Pritchett L. Estimating Wealth Effects without Expenditure Data - or Tears: Educational Enrollment in India. Washington DC: Development Economics Research Group, The World Bank; 1998.
25. Rohleder P, Eide AH, Swartz L, Ranchod C, Schneider M, Schür C. Gender differences in HIV knowledge and unsafe sexual behaviours among disabled people in South Africa. Disabil Rehabil 2012;34(7):605-610. https://doi.org/10. 3109/09638288.2011.599915

26. Tasnim S, Rahman A, Anamul Hoque FM. Patient's knowledge and attitude towards tuberculosis in an urban setting. Pulmonary Med 2012:352850. http:// dx.doi.org/10.1155/2012/352850

27. Wabiri N, Taffa N. Socio-economic inequality and HIV in South Africa. BMC Public Health 2013;13:1037. https://doi.org/10.1186/1471-2458-13-1037

28. Statistics South Africa (SSA). Mid-year population estimates 2017. Pretoria: SSA 2017. http://www.statssa.gov.za/publications/P0302/P03022017.pdf (accessed 4 December 2017)

29. Bekker LG, Hosek S. HIV and adolescents: Focus on young key populations. J Int AIDS Soc 2015;18(2Suppl 1):20076. https://doi.org/10.7448/IAS.18.2.20076

30. Joint United Nations Programme on HIV/AIDS (UNAIDS) 90:90:90: An ambitious treatment target to help end the AIDS epidemic. Geneva: UNAIDS; 2014. http://www.unaids.org/sites/default/files/media_asset/90-90-90_en.pdf (accessed 28 December 2017).

Accepted 20 April 2018. 